\title{
Efficient Determination of Accurate Force Fields for Porous Materials Using ab Initio Total Energy Calculations
}

\author{
Jihan Kim, ${ }^{* \dagger}{ }^{\dagger}$ Li-Chiang Lin, ${ }^{\ddagger}$ Kyuho Lee, ${ }^{\ddagger, \S}$ Jeffrey B. Neaton, ${ }^{\S}$ and Berend Smit ${ }^{\ddagger}, \|, \perp$ \\ ${ }^{\dagger}$ Department of Chemical and Biomolecular Engineering, Korea Advanced Institute of Science and Technology, 291 Daehak-ro \\ Yuseong-gu, Daejeon, Korea 305-710 \\ ${ }^{\ddagger}$ Department of Chemical and Biomolecular Engineering, University of California, Berkeley, Berkeley, California 94720, United States \\ ${ }^{\S}$ The Molecular Foundry, Lawrence Berkeley National Laboratory, Berkeley, California 94720, United States \\ "Department of Chemistry, University of California, Berkeley, Berkeley, California 94720, United States \\ ${ }^{\perp}$ Materials Sciences Division, Lawrence Berkeley National Laboratory, Berkeley, California 94720, United States
}

Supporting Information

\begin{abstract}
Accurate characterization of porous materials is essential for understanding material properties and evaluating their performance for potential applications. In general, any methodology that entails developing an accurate classical force field is computationally expensive as it requires a large number of quantum mechanical nonempirical calculations. In order to expedite such calculations without sacrificing too much accuracy, we have developed a systematic procedure where,

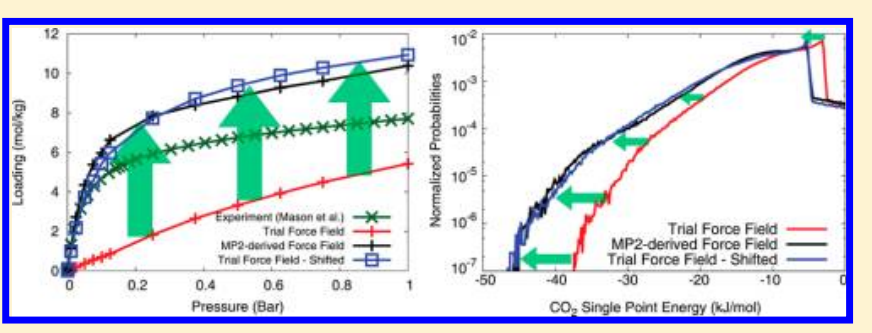
starting from an initial trial force field, accurate adsorption isotherms of porous materials can be obtained at low computational cost. Specifically, the procedure involves correcting singlepoint energy values sampled from the trial force field in grand canonical Monte Carlo simulations from few quantum mechanical calculations. We demonstrate that the methodology yields accurate adsorption data in diverse selection of guest molecules in porous materials such as $\mathrm{CH}_{4}$ and $\mathrm{CO}_{2}$ in zeolites (i.e., MFI, LTA, WEI, RHO, SOD, FAU, RWY, and ABW) and CO in metalorganic frameworks (i.e., M-MOF-74 with $\mathrm{M}=\mathrm{Mg}, \mathrm{Fe}$ ). Furthermore, we use our corrected force fields to predict the adsorption properties of $\mathrm{N}_{2}$ in V-MOF-74 and Ti-MOF-74, which are two materials that have yet to be synthesized experimentally. We anticipate that this methodology will be useful in accurately characterizing a given porous material in the absence of a reliable force field as well as for efficiently screening a large number of porous materials.
\end{abstract}

\section{INTRODUCTION}

Porous materials contain pores with length scales on the order of few angstroms that allow selective adsorption of guest molecules. Because of the large internal surface area and the enhanced adsorption capacities, porous materials are seen as promising candidates for many energy-related applications such as gas storage/separation and catalysis. ${ }^{1-4}$ In particular, there has been a growing amount of interest among the scientific community in a class of porous materials called metal-organic frameworks (MOFs), which are crystalline structures that consist of metal atoms and organic ligands. ${ }^{5-7}$ Because of a wide selection of choices available in the metal atoms, ligand types, and the topology of the structures, thousands of distinct MOFs have been synthesized, and the number of potential, hypothetical structures that can be synthesized is theoretically infinite. As the total number of experimentally synthesized MOFs continues to increase at a rapid pace, the ability to predict material properties prior to synthesis becomes critical for any rational MOF design. In particular, accurate characterization of the adsorption properties of porous materials becomes very important as it ties in directly with the storage and separation capabilities of the MOFs. In this regard, the experimental adsorption isotherm data provide a direct way to test the veracity of a given computational model as there should in principle be good agreement between the simulated and the experimental isotherm results upon adopting a correct model. From a computational perspective, accurate prediction of adsorption properties depends on the reliability of the force fields used to model the system in question. In the past, there have been several studies on large-scale screening of porous materials, but most of these works involved generic force fields, which may not yield accurate data for a large number of diverse structures. $^{8-14}$ In theory, one can bypass the force fields and instead conduct exclusively $a b$ initio quantum mechanical calculations. Unfortunately, this approach is time-consuming as quantum mechanical total energy calculations are very expensive, and the number of calculations needed to accurately characterize most of the systems remains large.

In order to improve computational efficiency, many efforts have focused on developing accurate force fields for porous

Received: September 23, 2013

Revised: January 13, 2014

Published: January 15, 2014 
materials. Fang et al. used hundreds of quantum mechanical calculations based on ab initio density functional theory (DFT) and the DFT-D2 and the DFT/CC approach to compute accurate force fields for pure-silica zeolites and aluminosilica zeolites, respectively. ${ }^{15,16} \mathrm{McD}$ aniel et al. developed force fields based on symmetry-adapted perturbation theory for $\mathrm{CO}_{2}$ adsorption in zeolitic imidazolate frameworks (ZIFs) and demonstrated transferability for selected ZIF structures. ${ }^{17}$ Han et al. utilized high-level ab initio calculations to develop accurate dispersive force fields for $\mathrm{CO}_{2}$ uptake in multivariate metal-organic framework structures. ${ }^{18}$ Dzubak et al. developed a technique where single-point MP2 energy calculations were conducted along given paths that were well-designed to probe the pairwise short-range interactions to derive the force field parameters. ${ }^{19}$ This approach yielded good agreement between the simulated and the experimental isotherm data in both open metal site MOF such as Mg-MOF-74 as well as an archetypical MOF such as MOF-5. Chen et al. conducted similar work on the Mg-MOF-74 structure where they used a multiobjective genetic algorithm to accurately fit over a thousand of single point energies, which resulted in good agreement with the experimental isotherms as well. ${ }^{20}$ However, the aforementioned methods may involve a large number of quantum mechanical calculations, which imposes great difficulties in extensively implementing these methods in large-scale screening applications. Sauer and co-workers have developed an alternative approach, in which high-level quantum mechanical methods are used to obtain binding energies at a small set of predefined adsorption sites. Sauer and co-workers have used this approach to model $\mathrm{CH}_{4}$ adsorption in Mg-MOF-74 structure. ${ }^{21}$

In this work, we introduce a simple and efficient method that leads to accurate prediction of adsorption properties for various porous materials in absence of a reliable fore field. Compared to $\mathrm{ab}$ initio methods, a small amount of CPU time is required to compute an adsorption isotherm using conventional grand canonical Monte Carlo simulations. For example, using one of the generic force fields such as the UFF or Dreiding, we can easily compute an adsorption isotherm curve at the conditions of interest. We take advantage of the fact that although such force fields might be initially inaccurate, they lead to a qualitatively correct description about the location of the adsorption sites as well as the shape of the channels and the pores. Consequently, one can start from an inaccurate force field and utilizing just a few quantum mechanical calculations converge to a model that leads to the correct adsorption properties. In this respect, our work is similar to the methodology proposed by Sauer and co-workers. ${ }^{21}$ They constructed a multisite Langmuir isotherm as an "estimate" of the isotherm and used quantum mechanical calculations to obtain the parameters for this model. Comparatively, the advantage of our methodology is that we do not have to assume prior knowledge on the number and location of the adsorption sites and that it automatically includes guest-guest interactions.

The methodology introduced in this paper will be valuable in characterizing hypothetical porous materials where reliable force fields do not exist. On top of this, the methodology remains relatively unchanged regardless of the simulated material, making it ideal for large-scale screening. In the rest of the paper, the detail behind the methodology and the simulation results gathered from various different systems will be shown to demonstrate its accuracy as well as its transferability.

\section{RESULTS AND DISCUSSION}

A. $\mathrm{CO}_{2}$ in Mg-MOF-74. To describe the methodology, we introduce a test system of $\mathrm{CO}_{2}$ inside $\mathrm{Mg}$-MOF-74, which has shown to be promising for carbon capture due to the presence of unsaturated open-metal sites. ${ }^{22-26}$ The main reason for choosing this system is as follows. In the past, we have developed an MP2-derived force field in this same system, demonstrating excellent agreement between the simulation and the experimental data. Accordingly, for our purpose, we can regard the MP2-derived force field as providing a high-quality reference result and subsequently gather millions of single point energy values at low computational cost, which can help better illustrate our methodology. In practice, for a structure where the force field is unknown, we will not have this information at our disposal. However, this does not pose a problem as our algorithm does not rely on collection of millions of single point energy calculations prior to the simulation. All of the classical molecular simulation data obtained from this work came from our in-house developed graphics processing units (GPU) code, ${ }^{27}$ where the algorithm detail is described elsewhere. ${ }^{27}$

For the initial step, a trial force field that can reasonably describe the system of interest should be chosen. In our case, the UFF and TraPPE force field parameters are selected to model the Mg-MOF-74 framework atoms and the $\mathrm{CO}_{2}$ molecule, respectively, as these are commonly used force fields adopted by the community. In practice, our methodology should converge to the same model regardless of the initial force field choice. We will revisit this point later on in the paper.

In general, the UFF force field provides the parameters for the short-range repulsions and dispersive interactions, while the Coulomb interactions follow from the charge distribution on the guest atoms and the MOFs. For the DFT-computed MgMOF-74 framework, point charges are obtained from the relaxed Mg-MOF-74 ab initio structure using the REPEAT scheme. $^{28}$ The Mg-MOF-74 structure was taken from our previous work, ${ }^{19}$ in which the lattice vectors and atomic positions are fully relaxed using the DFT package Quantum Espresso. $^{29}$ The REPEAT scheme iteratively finds a set of framework point charges that minimizes the norm difference between the electrostatic potential of atomic charge model and the quantum mechanical self-consistent electrostatic potential. Because the REPEAT scheme incurs very little computational cost compared to the entire structure relaxation procedure, it is worthwhile to obtain these accurate point charge values for our methodology since the structures need to be relaxed before the classical simulation in most cases and especially for these openmetal site structures. Subsequently, the only difference between the MP2-derived force field and the trial force field comes from the short-range repulsion and the dispersive interaction energies between the $\mathrm{CO}_{2}$ and the framework atoms. However, this discrepancy leads to drastically different adsorption data as the two sets of force field yields $K_{\mathrm{H}}=1.13 \times 10^{-3} \mathrm{~mol} /(\mathrm{kg} \mathrm{Pa})$ (MP2-derived force field) and $K_{\mathrm{H}}=7.2 \times 10^{-5} \mathrm{~mol} /(\mathrm{kg} \mathrm{Pa})$ (UFF) at $T=313 \mathrm{~K}$, resulting in two completely different adsorption isotherm curves as shown in Figure 1a. Comparison between the simulated and the experimental data verifies that the MP2-derived force field leads to a better fit with the previous experiments. ${ }^{30}$ In our calculations, we assume that all metal sites are active without any defects present in the structure. However, it has been shown experimentally that about $20 \%$ of metal sites are inaccessible, which may be due to 


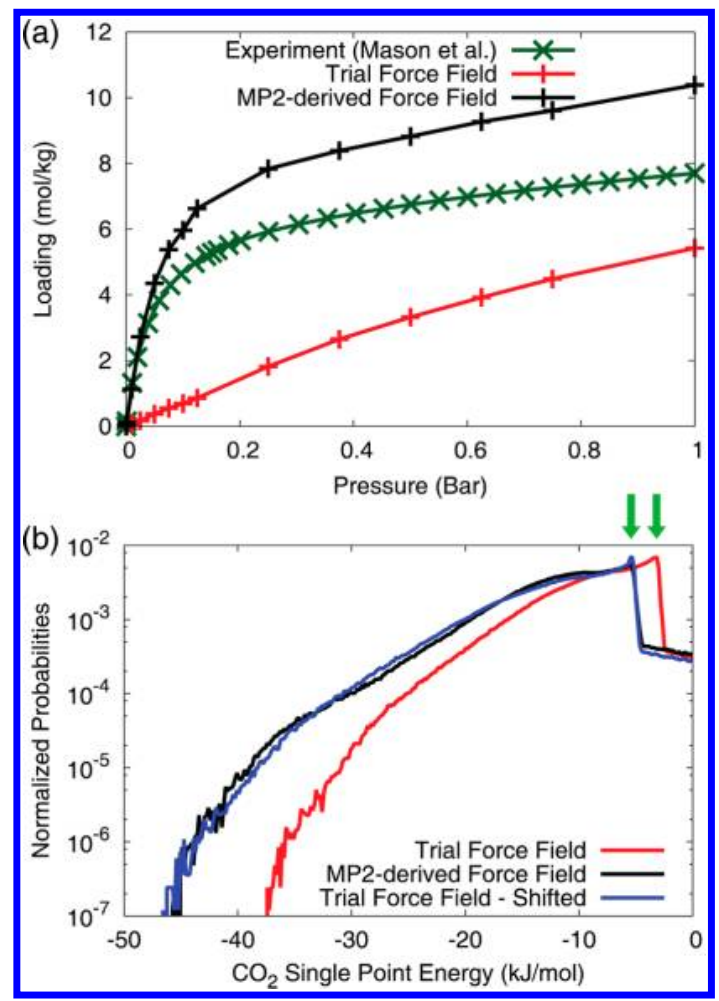

Figure 1. (a) Experimental (green) and simulated (black: MP2derived force field; red: UFF) adsorption isotherms for $\mathrm{CO}_{2}$ in $\mathrm{Mg}$ MOF-74 structure at $T=313 \mathrm{~K}$. (b) Distribution of the $\mathrm{CO}_{2}$ single point energy (black: MP2-derived force field; red: UFF; blue: UFF, shifted) values based on 8 million randomized insertions. The green arrows indicate the location of maximum points within the single point energy distribution, corresponding spatially to the pore centers in the Mg-MOF-74 structure.

pore blocking or activation issue. ${ }^{24}$ Accordingly, the MP2derived computational model does not account for this imperfection and thus overestimates the adsorption data with the discrepancy becoming more pronounced at $P>0.1$ bar. To understand the source behind the difference between the adsorption data obtained MP2-derived force field and the trial (UFF) force field, the single point energy values are collected from random sampling. Specifically, over 8 million insertion moves are conducted on a preconstructed grid with a mesh size of $0.1 \AA$, superimposed on top of the unit cell simulation volume. At each grid point, the single point energy calculation of a randomized $\mathrm{CO}_{2}$ configuration is computed and then tabulated to obtain the normalized probability distribution shown in Figure 1b. As can be seen from Figure 1b, the shapes of the two curves look qualitatively similar, hinting that the trial force field manages to capture the potential energy surface of the guest-framework interactions. This qualitative agreement has allowed us to understand the dynamic properties of $\mathrm{CO}_{2}$ inside Mg-MOF-74 in our previous work with the trial force field (i.e., UFF).$^{31}$ Accordingly, minor corrections in the trial force field that artificially shift the single point curve toward the MP2-derived force field can lead to the correct Henry coefficient value. The total adsorption isotherm fit outside of the Henry regime will also depend on the guest-guest (or, in this case, $\mathrm{CO}_{2}-\mathrm{CO}_{2}$ ) interactions, which is modeled well by the TraPPE force field. The TraPPE force field has been shown to reproduce the $\mathrm{CO}_{2}$ vapor-liquid equilibria that are dominated by intermolecule interaction of the $\mathrm{CO}_{2}$ molecules.
In Figure $1 b$, the minimum single point energy region corresponds to the binding energies for the two force fields. In this particular instance, the two $\mathrm{CO}_{2}$ binding energies are $E_{\text {reference,binding }}=-46.22 \mathrm{~kJ} / \mathrm{mol}$ (MP2-based force field) and $E_{\text {trial,binding }}=-37.41 \mathrm{~kJ} / \mathrm{mol}$ (trial force field), leading to a difference of $\Delta E_{\text {binding }}=8.81 \mathrm{~kJ} / \mathrm{mol}(=1059 \mathrm{~K})$. Accordingly, one can shift all of the energy values obtained during the molecular simulation using the trial force field by $\Delta E_{\text {binding }}$ to obtain a better fit to the MP2-derived energy distribution near the binding energy region. Simple math indicates that $K_{\mathrm{H} \text {,shifted }}$ $=7.2 \times 10^{-5} \times \exp (-1059 \mathrm{~K} / 313 \mathrm{~K})=2.12 \times 10^{-3} \mathrm{~mol} /(\mathrm{kg}$ $\mathrm{Pa})$, moving closer to the MP2-derived $K_{\mathrm{H}}$. In practice, the two binding energies, $E_{\text {reference,binding }}$ and $E_{\text {trial,binding }}$, can be obtained from the DFT calculations and the classical canonical Monte Carlo simulations near $T=0 \mathrm{~K}$, respectively. And thus remarkably, with a single quantum mechanical binding energy calculation, one can potentially obtain adsorption properties much closer to the reference result.

In principle, additional single point energy calculations can be utilized from the MP2-derived force field to converge even closer to the correct energy distribution curve. In practice, this would entail conducting more quantum mechanical calculations, which would enhance accuracy at additional computational cost. In general, there is not a clear-cut recipe on selecting the spatial location of a guest molecule for these single point energy quantum mechanical calculations. An idea suggested in this work is to sample near a local maximum region of the energy distribution curve at higher energies values and shift the trial force field to match these data points (e.g., region indicated by the two arrows in Figure $1 \mathrm{~b}$ ).

This secondary shift is conducted near the local, normalized probability distribution maximum values, located between -6 and $-3 \mathrm{~kJ} / \mathrm{mol}$ in Figure $1 \mathrm{~b}$. Spatially, the local maximum region corresponds to the center of the one-dimensional channel in the Mg-MOF-74 structure. The sudden drop in the curves appears for both force fields as it is not possible to move further away from the $\mathrm{Mg}$ metal atoms at the pore center. Unsurprisingly, $\Delta E_{\text {center }}=E_{\text {trial,center }}-E_{\text {reference,center }}=(-3.33)-$ $(-5.49)=2.16 \mathrm{~kJ} / \mathrm{mol}<\Delta E_{\text {binding }}=8.81 \mathrm{~kJ} / \mathrm{mol}$, as the guest host van der Waals interaction weakens further away from the open metal sites. In other words, the trial force field does a better job of capturing the correct adsorption properties far away from the strong binding adsorption sites. In practice, one could forego conducting additional quantum mechanical calculations at the pore center and assume that $E_{\text {reference,center }}$ $=E_{\text {trial,center }}=-3.33 \mathrm{~kJ} / \mathrm{mol}$ in order to reduce the large computational cost incurred while retaining similar level of accuracy.

Taking account these two shifts, all of the energy values less than $E_{\text {reference,center }}$ are shifted by $\Delta E_{\text {center }}$, and at the binding energy, the energy is shifted by $\Delta E_{\text {binding. }}$. In between the two points, we opt for a linearized shift such that $\Delta E_{\text {shift }}$ increases linearly from $E_{\text {trial,center }}$ and $E_{\text {trial,binding. The resulting shifted }}$ curve is displayed in Figure $1 \mathrm{~b}$ in blue, and it agrees very well with the MP2-derived distribution. The resulting $\mathrm{CO}_{2} K_{\mathrm{H}}=$ $1.00 \times 10^{-3} \mathrm{~mol} /(\mathrm{kg} \mathrm{Pa})$, which is only $13.0 \%$ less than the MP2-derived force field $K_{\mathrm{H}}=1.13 \times 10^{-3} \mathrm{~mol} /(\mathrm{kg} \mathrm{Pa})$. The $\mathrm{CO}_{2} K_{\mathrm{H}}$ was computed by shifting the energies by an appropriate amount during the Widom particle insertion moves. If we forego the secondary shift at the pore center and assume $E_{\text {reference,center }}=E_{\text {trial,center, }}$ the resulting linearized shift yields $\mathrm{CO}_{2} \mathrm{~K}_{\mathrm{H}}=8.18 \times 10^{-4} \mathrm{~mol} /(\mathrm{kg} \mathrm{Pa})$, which is $38 \%$ less than the MP2-derived force field $K_{\mathrm{H}}$ but still a reasonably 
good prediction. And using grand canonical Monte Carlo simulations, we observe that there is good agreement at high pressure regions as well ( 1 bar: $11.27 \mathrm{vs} 10.22 \mathrm{~mol} / \mathrm{kg}$ ) and (10 bar: $14.31 \mathrm{~mol} / \mathrm{kg}$ vs $13.65 \mathrm{~mol} / \mathrm{kg}$ ), with the former being the reference result and the latter the shifted result neglecting the secondary shift.

For the purpose of illustration, the binding energy of $\mathrm{CO}_{2}$ inside Mg-MOF-74 was computed directly from the MP2derived force field, and the value was regarded as an "exact" baseline. However, the binding energy values obtained from DFT as well as other wave function based quantum mechanical methods contain several uncertainties such as the choice of functional for the dispersive energies on the DFT level and basis sets. Thus, it is important to evaluate the sensitivity of the algorithm to the binding energy values. Our results are summarized in the Supporting Information where computational results for both the $\mathrm{CO}_{2} K_{\mathrm{H}}$ and the full isotherms are reported at varying $\mathrm{CO}_{2}$ binding energies. Overall, the stability analysis illustrates the importance of obtaining accurate quantum mechanical binding energies for this system in the context of our methodology. As expected, when the binding energies become inaccurate, the adsorption properties deviate from the reference results.

Next, the effect of the initial choice in the trial force field is analyzed. An algorithm in which the final adsorption data are completely independent of the choice in the trial force field is ideal. In order to conduct the sensitivity analysis, the point charges in the $\mathrm{CO}_{2}$ molecule were changed from their initial values of $q_{\mathrm{C}, \mathrm{CO}_{2}}=+0.7, q_{\mathrm{O}, \mathrm{CO}_{2}}=-0.35$ to $q_{\mathrm{C}, \mathrm{CO}_{2}}=+0.6,+0.5$, +0.4 , and +0.3 (with corresponding changes in $q_{\mathrm{O}, \mathrm{CO}_{2}}$ to ensure neutrality). The resulting single point energy distributions are shown in Figure 2a. The results here indicate that decreasing $q_{\mathrm{C}, \mathrm{CO}_{2}}$ effectively reduces the energy spread (i.e., energy difference between the minima point and the local maximum point in the curve) within the distribution. This is reasonable as imposing stronger electrostatic interactions cause sharper peaks in the minima energy. Moreover, the local peak in the distributions corresponds to the center of the pore region where the sum of the electrostatic interaction contributions is small, thus making it independent of charge. Accordingly, for smaller $q_{\mathrm{C}, \mathrm{CO}_{2}}$ in the initial trial force field, the subsequent shift will cause a greater overestimation in the infinite dilution adsorption property as there will be proportionally more single point energies near the binding energy region when the energy spread is small. Mathematically, this effect can be visualized upon subtracting the shifted trial force energy distributions from the MP2-derived distribution (Figure 2b). As can be seen from Figure $2 b$, for smaller $q_{\mathrm{C}, \mathrm{CO}_{2}}$, the overestimate from the trial force field increases between -35 and $-18 \mathrm{~kJ} / \mathrm{mol}$. Since the low-energy region contributes mostly to the infinite dilution adsorption properties, it is predicted that the discrepancy between the Henry coefficient values computed from the MP2derived force field and the trial force field will increase for decreasing $q_{\mathrm{C}, \mathrm{CO}_{2}}$. This is supported in our calculations where for $q_{\mathrm{C}, \mathrm{CO}_{2}}=+0.6,+0.5,+0.4$, and +0.3 , the $\mathrm{CO}_{2} K_{\mathrm{H}}=9.5 \times$ $10^{-4}, 1.1 \times 10^{-3}, 1.43 \times 10^{-3}$, and $1.69 \times 10^{-3} \mathrm{~mol} /(\mathrm{kg} \mathrm{Pa})$, respectively. The resulting data show that even for $q_{\mathrm{C}_{,} \mathrm{CO}_{2}}=$ +0.3 , which is most different from the original charge value of $q_{\mathrm{C}, \mathrm{CO}_{2}}=+0.7$, the shifted correction does an accurate job of estimating the MP2-derived $\mathrm{CO}_{2} K_{\mathrm{H}}$ value. However, the

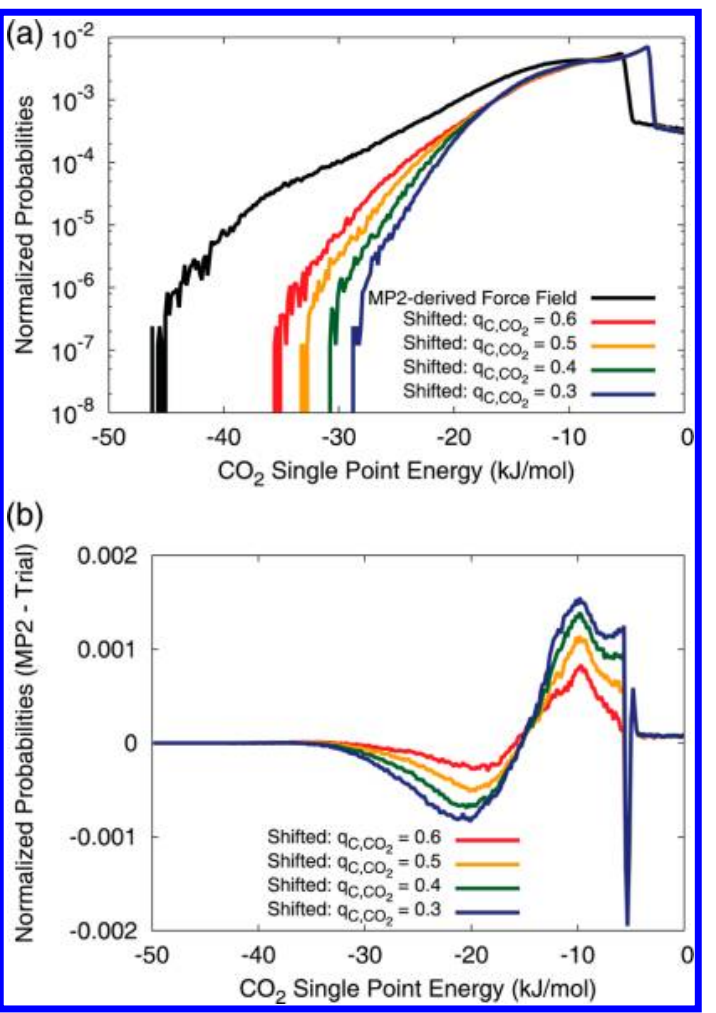

Figure 2. (a) $\mathrm{CO}_{2}$ single point energy distributions for MP2-derived force field (black) and UFF for $q_{\mathrm{C}, \mathrm{CO}_{2}}=+0.6,+0.5,+0.4$, and +0.3 . (b) The difference between the energy distributions of MP2-derived force field (black line in (a)) and the shifted UFF (shifted lines in (a)) for $q_{\mathrm{C}, \mathrm{CO}_{2}}=+0.6,+0.5,+0.4$, and +0.3 .

results here underscore the importance of judicious selection of the initial force field and charge parameters. We also note from Figure $2 \mathrm{~b}$ that there is a spike at $-5 \mathrm{~kJ} / \mathrm{mol}$ near the $E_{\text {center }}$ region. Upon conducting shifts, there are binning mismatches between the reference distribution and the shifted one due to a sharp discontinuity at the point, creating a numerical artifact. However, this does not affect any of the conclusions drawn from our analysis.

Finally, we would like to point out that there can be other methods to correct the energy distribution instead of conducting shifts. An example of this would be to correct based on the scaling factors (e.g., ratio between the reference and the trial energies). However, in this case there can be problems where the scaling factors at the secondary sites become much larger than the one at the primary site, potentially resulting in significant overcorrection. In the future, we plan to look at other methodologies that can perhaps improve upon the current methodology adopted in this paper.

B. $\mathrm{CO}_{2}$ in Fe-MOF-74 and $\mathrm{N}_{2}$ in $\mathrm{V}$,Ti-MOF-74. In the previous section, the reference result that was used for comparison purposes was derived from classical molecular simulation data using an MP2-derived force field., However, the utility of this methodology hinges on being able to predict or to reproduce experimental data in absence of a good force field. Thus, we proceed to select two systems: (1) $\mathrm{CO}_{2}$ in Fe-MOF74 and (2) $\mathrm{N}_{2}$ in V-MOF-74 and Ti-MOF-74, corresponding to cases where (1) there are experimental data in the literature ${ }^{32}$ and (2) there are neither experimental nor simulated isotherm data published anywhere. 
For this analysis, the binding energies were computed using DFT with a vdW-DF2 functional ${ }^{33,34}$ with on-site Hubbard U corrections $^{35}$ for metal d electrons as implemented in VASP. ${ }^{36}$ The computed binding energies for $\mathrm{CO}_{2}$ in Fe-MOF-74, $\mathrm{N}_{2}$ in V-MOF-74, and Ti-MOF-74 are $-41,-56$, and $-59 \mathrm{~kJ} / \mathrm{mol}$, respectively. We used a plane-wave energy cutoff of $1000 \mathrm{eV}$ and projector augmented wave potentials. The Brillouin zone was sampled at the $\Gamma$-point. All calculations are spin polarized. For both Fe-MOF-74 and V-MOF-74, the initial on-site magnetic moments of metal cations were set to their highspin state according to Hund's rule in a weak-field ligand environment and eventually converged to the same high-spin state at the end of the self-consistent electronic iteration. We obtained ferromagnetic ordering along the metal oxide chain direction and antiferromagnetic between the chains for FeMOF-74, and we assumed the same ordering for V-MOF-74. For bare MOFs we used a triclinic primitive unit cell containing 54 atoms including 6 metal centers and simultaneously optimized the lattice vectors and the atomic positions in the unit cell with variable cell dynamics with $\mathrm{PBE}+\mathrm{U}$. The calculated lattice constants of Fe-MOF-74 for the conventional hexagonal unit cell are $a=b=26.47 \AA$ and $c=6.97 \AA$, which are in good agreement with experiment to within $1.7 \%$. Then the MOF structure is assumed to be rigid while all atomic positions of the guest molecules in the MOF are fully relaxed by using vdW-DF2+U until the residual forces are smaller than $0.01 \mathrm{eV} / \AA$. For the secondary correction at the center of the pore, we utilize the $E_{\text {reference,center }}=E_{\text {trial,center }}$ where $E_{\text {trial,center }}$ is computed from the trial force field.

Given that the binding energies directly come from quantum mechanical calculations with Born-Oppenheimer approximation where nuclear quantum fluctuations are not included, the zero-point energy contributions to the binding energy need to be computed and subtracted from the shift. The zero-point energy corrections computed for $\mathrm{CO}_{2}$ in Fe-MOF-74 and $\mathrm{N}_{2}$ in V-MOF-74 and Ti-MOF-74 are 1.8, 4.1, and $3.9 \mathrm{~kJ} / \mathrm{mol}$, respectively. The zero-point energies are calculated using DFT vibrational frequencies at the harmonic level (for details see ref 37).

The simulated adsorption isotherm data for the two systems are shown in Figures $3 \mathrm{a}$ and $4 \mathrm{~b}$. Using the UFF force field, the $\mathrm{CO}_{2}$ TraPPE model, and the point framework charges obtained from the REPEAT scheme, the computed binding energies are $-34.23,-18.58$, and $-18.7 \mathrm{~kJ} / \mathrm{mol}$ for $\mathrm{CO}_{2}$ in Fe-MOF-74 and $\mathrm{N}_{2}$ in V-MOF-74 and Ti-MOF-74. For the secondary shift, the center of the pore energy values was assumed to be the same between the trial force field and the DFT results. Figure 3a shows very good agreement between the experimental and the simulated (shifted) isotherm data for $\mathrm{CO}_{2}$ in Fe-MOF-74 at $\mathrm{T}$ $=298 \mathrm{~K}$. The overestimation of $\mathrm{CO}_{2}$ uptake at relatively high pressure values might be due to the presence of inactive metal sites in the conducted experiments. For the $\mathrm{N}_{2}$ isotherm data, because the UFF binding energy values are very different from those computed from the DFT method, the disparity between the UFF and the shifted isotherms is significantly larger (Figure 3b) for both V-MOF-74 and Ti-MOF-74. Lee et al. have shown that this exceptionally strong binding of $\mathrm{N}_{2}$ to V-MOF-74 is due to the subtle interplay of several interactions. ${ }^{37}$ In practice, our methodology can be used to compute all of the isotherms for various guest molecules in the M-MOF-74 structures for different metal atoms. Conceivably, this will allow us to screen the materials and identify the optimal open metal site MOF structures for various different applications of interest.

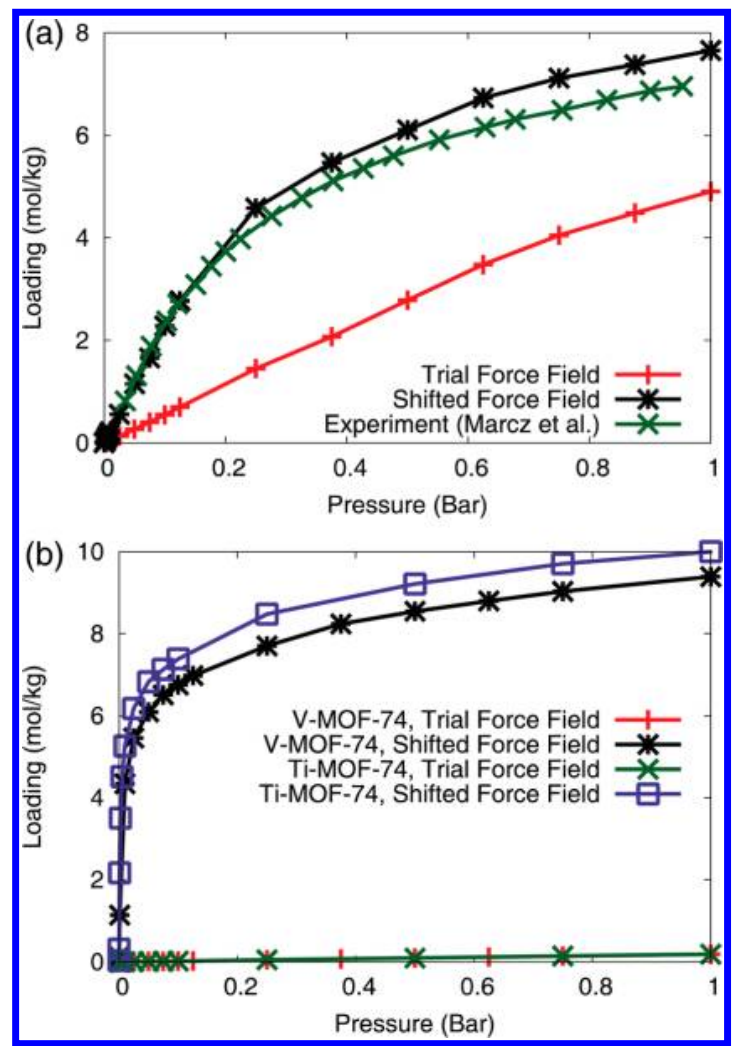

Figure 3. Adsorption isotherm for (a) $\mathrm{CO}_{2}$ in Fe-MOF-74 at $T=298$ $\mathrm{K}$ and (b) $\mathrm{N}_{2}$ in V-MOF-74 at $T=313 \mathrm{~K}$. The experimental data for $\mathrm{N}_{2}$ in V-MOF-74 do not exist and thus were excluded from the figure.

C. $\mathrm{CH}_{4}$ and $\mathrm{CO}_{2}$ in Zeolites. In the previous systems, the adsorption is dominated by the interactions with the open metal sites. To test our method for a system that is less dominated by strong binding sites, $\mathrm{CH}_{4}$ and $\mathrm{CO}_{2}$ inside zeolite structures are analyzed next. In particular, eight common puresilica zeolite structures in the IZA database (i.e., MFI, LTA, WEI, RHO, SOD, FAU, RWY, and ABW) are chosen to demonstrate the transferability within the class of zeolite materials. The crystal structures for each of these structures can be found from an online IZA database. ${ }^{38}$ In this analysis, the force field and charge parameter values taken from Garcia-Perez et al. ${ }^{39}$ were selected as the true, reference model as it has been demonstrated to reproduce existing experimental data for pure silica zeolite structures. ${ }^{39}$

As a baseline, the energy distribution curves that arise from millions of $\mathrm{CH}_{4}$ and $\mathrm{CO}_{2}$ single point energy calculations and the $\mathrm{CH}_{4}$ and $\mathrm{CO}_{2} \mathrm{~K}_{\mathrm{H}}$ computed from the Garcia-Perez force field were treated as being accurate. The $\mathrm{CH}_{4}$ energy distributions for all eight zeolites are illustrated in the Supporting Information. The distributions that emerge for different zeolite structures reflect the variety of pore topologies that exist within the class of zeolite materials. For example, in zeolite SOD, the single point energy has a local maximum near the binding energy region. Also, in structures like ABW, WEI, and SOD, there does not seem to be a distinct maximum peak that is found from the open-metal site structures. In light of this diversity, we opt to choose two different shifting strategies: (1) constant uniform energy shift, $\Delta E_{\text {binding }}$, for all single point energy values and (2) linear shift with the secondary match being set to $E=0 \mathrm{~kJ} / \mathrm{mol}$. The latter value was chosen as an 

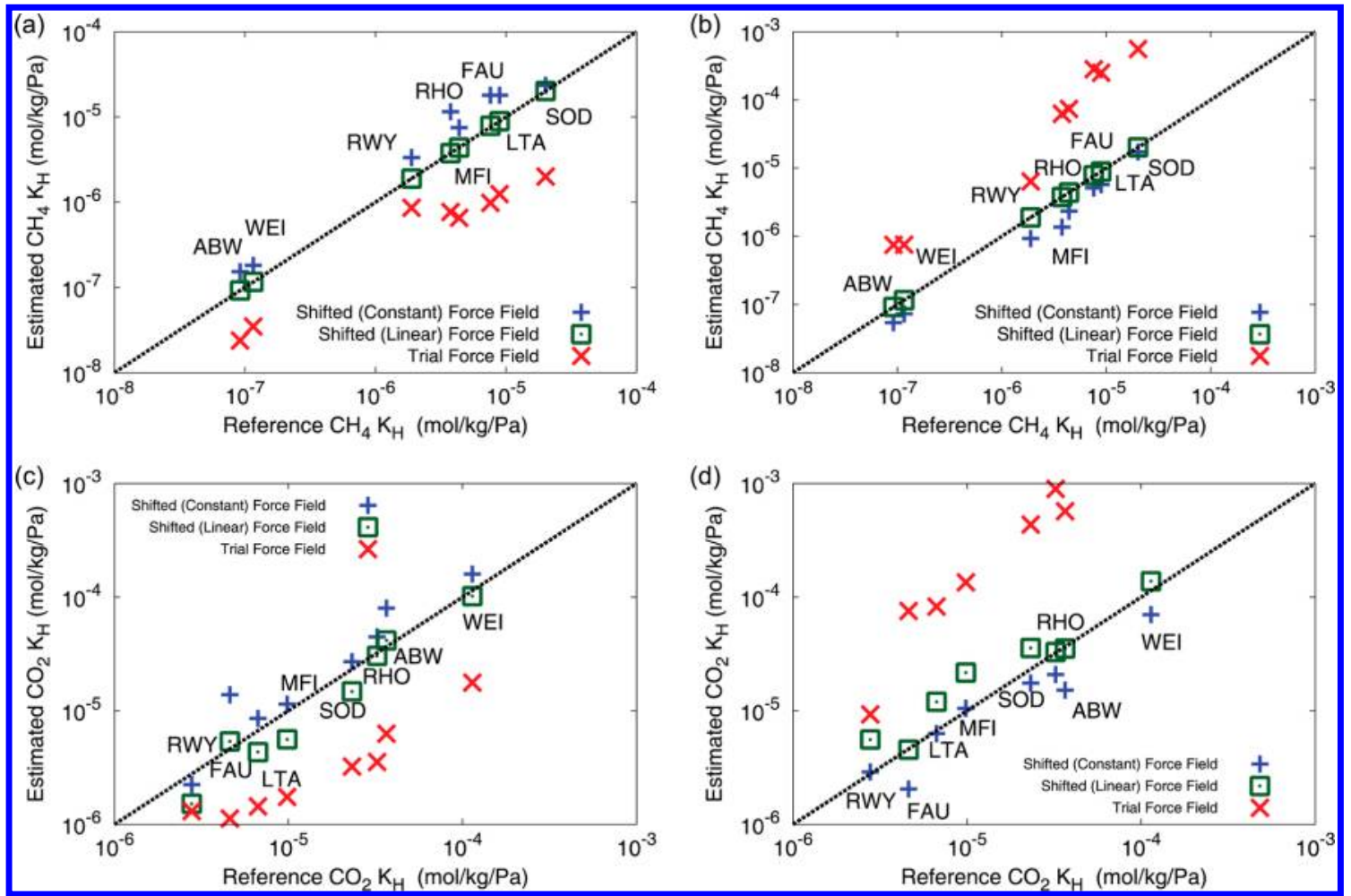

Figure 4. Estimated and the reference $\mathrm{CH}_{4}(\mathrm{a}, \mathrm{b})$ and $\mathrm{CO}_{2}(\mathrm{c}, \mathrm{d}) \mathrm{K}_{\mathrm{H}}$ for zeolites MFI, LTA, WEI, RHO, SOD, FAU, RWY, and ABW. The dashed line is where the predicted and the reference data are equal to one another. The Lennard-Jones parameter for oxygen are $(\mathrm{a}, \mathrm{c}) \varepsilon_{\text {oxygen }}=44.68 \mathrm{~K}$ and (b, d) $\varepsilon_{\text {oxygen }}=178.72 \mathrm{~K}$. For the constant shift, all of the energy values are shifted by a constant amount equal to the difference between the binding energies. For the linear shift, $0 \mathrm{~kJ} / \mathrm{mol}$ is taken as the point for which there is zero shift for larger energy values.

adequate reference point to smooth out any overshifting from a constant uniform energy shift.

For the two initial sets of trial force fields, the pairwise potential depth in the Lennard-Jones parameter $\varepsilon_{\text {oxygen }}$ of the zeolite framework atom is increased by $2 \times$ for and decreased by $2 \times$ for the other from its initial value, $\varepsilon_{\text {oxygen }}=89.36 \mathrm{~K}$. Effectively, the two starting force fields respectively overestimate and underestimate the correct adsorption data. In Figure 4, three different sets of data are plotted against the reference $\mathrm{CH}_{4} K_{\mathrm{H}}(\mathrm{a}, \mathrm{b})$ and $\mathrm{CO}_{2} K_{\mathrm{H}}(\mathrm{c}, \mathrm{d})$ data ( $x$-axis) at $\mathrm{T}=$ $313 \mathrm{~K}$ for the eight selected zeolite structures: (1) $K_{\mathrm{H}}$ values (" $X$ " data points) obtained from force field with the "wrong" initial force field, (2) $K_{\mathrm{H}}$ values ("+" data points) obtained from constant uniform shift force field, and (3) $K_{\mathrm{H}}$ values (" $\square$ " data points) obtained from linear shift force field. In Figure $4 a, c$ data, the starting trial force field parameter has $\varepsilon_{\text {oxygen }}=44.68$ $\mathrm{K}$, while in Figure $4 \mathrm{~b}, \mathrm{~d}$ the starting trial force field parameter has $\varepsilon_{\text {oxygen }}=178.72 \mathrm{~K}$. The dashed line indicates the region where the $K_{\mathrm{H}}$ values determined from the corrected force field and the reference force field are equal to one another. Figure 4 indicates that the wrong initial data set (" $X$ " data points) underestimates the reference $K_{\mathrm{H}}$ result for smaller $\varepsilon_{\text {oxygen }}$ (Figure $4 \mathrm{a}, \mathrm{c}$ ) and overestimates the reference $K_{\mathrm{H}}$ result for larger $\varepsilon_{\text {oxygen }}$ (Figure $4 \mathrm{~b}, \mathrm{~d}$ ), which is reasonable given that $\varepsilon_{\text {oxygen }}$ has positive correlation with binding energy values.

The data points generated from using a constant, uniform shift force field is shown as "+" data points in Figure 4. In general, because all of the energy curves are shifted by the same amount of $\Delta E_{\text {binding, }}$ there is overestimation of adsorption property with smaller $\varepsilon_{\text {oxygen }}$ (i.e., data points are above the dashed line in Figure $4 \mathrm{a}, \mathrm{c}$ ) and underestimation with larger $\varepsilon_{\text {oxygen }}$ (i.e., data points are below the dashed line in Figure
$4 \mathrm{~b}, \mathrm{~d})$. However, this trend does not hold for all data points as can be seen for example, in the case of zeolite RWY (Figure 4c). We attribute this to the three-site model of the $\mathrm{CO}_{2}$ that results in less predictability compared to the case for $\mathrm{CH}_{4}$. Overall, it can be seen that in few cases the constant shift results in a worse fit compared to the $K_{\mathrm{H}}$ data obtained from a wrong force field. However, all of the data points tend to collectively move toward the dashed line for the shift.

Finally, the data points generated from using a linear shift are shown as (" $\square$ " data points) in Figure 4. For $\mathrm{CH}_{4}$, the linear shift results in remarkably good match between the shifted and the reference data, demonstrating the importance of simultaneously shifting to the correct binding energy while tempering the correction to avoid overshifting. For $\mathrm{CO}_{2}$, it becomes less clear on whether the linear shift does a better job compared to the constant shift, which is not surprising given the presence of larger degree of freedom found for the $\mathrm{CO}_{2}$ molecule compared to $\mathrm{CH}_{4}$. Overall, the results here seem to indicate that the methodology does a very good job of retrieving the correct adsorption data in cases where the initial force field and reference force field differ only by the pairwise potential depth (i.e., $\varepsilon$ ) in the Lennard-Jones parameter.

Next, we move on to a case where the initial force field has a different $\sigma$ value in the Lennard-Jones parameters, where $\sigma$ is the distance at which the interparticle interaction goes to zero. Specifically, the $\sigma_{\text {oxygen }}$ was decreased from $\sigma_{\text {oxygen }}=3.21$ to 2.568 (i.e., $20 \%$ reduction) in the trial force field parameter. The resulting data are shown in Figure 5 with the symbols corresponding to the same force fields as in Figure 4.

Overall, for both $\mathrm{CH}_{4}$ and $\mathrm{CO}_{2}$, the agreement between the predicted and the reference results is worse for both sets of trial force fields (“+” and “ $\square$ ” data points). Because changes in $\sigma$ 


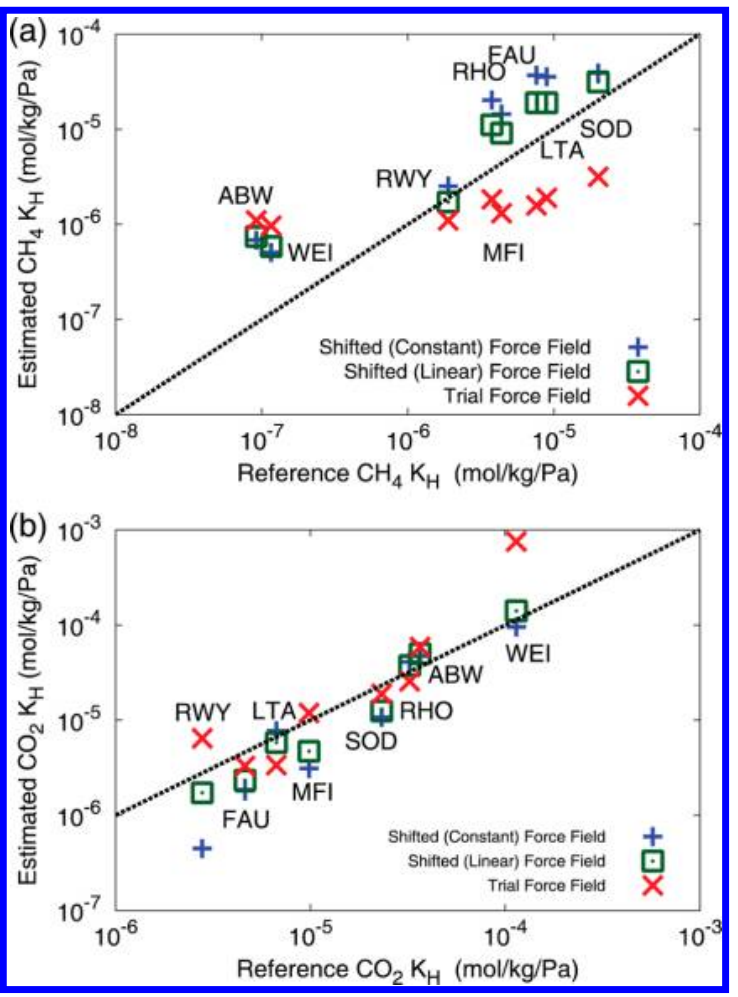

Figure 5. Estimated and the reference (a) $\mathrm{CH}_{4}$ and (b) $\mathrm{CO}_{2} \mathrm{~K}_{\mathrm{H}}$ for zeolites MFI, LTA, WEI, RHO, SOD, FAU, RWY, and ABW with a trial force field with $\sigma_{\text {oxygen }}=2.568$.

effectively change the binding distances, an overall shift in the single point energy values lead to relatively more unpredictable behavior compared to the situation where $\varepsilon$ was being changed. As a result, the selection in a trial force field that correctly matches the true $\sigma$ becomes more important as opposed to one that yields correct $\varepsilon$ in our methodology.

The predicted (from linear shift) and the reference $K_{\mathrm{H}}$ for all eight zeolites in Table 1 indicate that the structures with the worst $K_{\mathrm{H}}$ predictions are $\mathrm{ABW}$ and WEI. Geometric analysis using Zeo++, ${ }^{40}$ which are summarized in Table 1 , reveal that $\mathrm{ABW}$ and WEI have the smallest pore limiting diameter and the smallest maximum cavity diameter, thus leading to narrow channels in these structures. Subsequently, starting with an

Table 1. Estimated and the Reference $\mathrm{CH}_{4} \mathrm{~K}_{\mathrm{H}}$ for Zeolites MFI, LTA, WEI, RHO, SOD, FAU, RWY, and ABW with a Trial Force Field with $\sigma_{\text {oxygen }}=2.568^{a}$

\begin{tabular}{lccccc} 
& \multicolumn{2}{c}{$K_{\mathrm{H}}(\mathrm{mol} /(\mathrm{kg} \mathrm{Pa}))$} & & \\
\cline { 2 - 3 } & reference & linear shifted & & $\begin{array}{c}\text { pore limiting } \\
\text { diam }(\AA)\end{array}$ & $\begin{array}{c}\text { largest cavity } \\
\text { diam }(\AA)\end{array}$ \\
MFI & $4.4 \times 10^{-6}$ & $9.1 \times 10^{-6}$ & & 4.64 & 6.30 \\
LTA & $8.9 \times 10^{-6}$ & $1.9 \times 10^{-5}$ & & 4.15 & 10.99 \\
WEI & $1.2 \times 10^{-7}$ & $5.9 \times 10^{-7}$ & & 3.40 & 4.13 \\
RHO & $3.8 \times 10^{-6}$ & $1.1 \times 10^{-5}$ & & 4.00 & 10.37 \\
SOD & $2.0 \times 10^{-5}$ & $3.1 \times 10^{-5}$ & & 2.47 & 6.26 \\
FAU & $7.6 \times 10^{-6}$ & $1.9 \times 10^{-5}$ & & 7.29 & 11.18 \\
RWY & $1.9 \times 10^{-6}$ & $1.7 \times 10^{-6}$ & & 6.23 & 14.34 \\
ABW & $9.2 \times 10^{-8}$ & $7.4 \times 10^{-7}$ & & 3.45 & 4.18
\end{tabular}

${ }^{a}$ The pore limiting diameter and the largest cavity diameter obtained from Zeo++ are included. The boldface numbers indicate structures with small pores. incorrect $\sigma$ value leads to energy distribution that is completely different from the correct one as within these narrow pores. Overall, the sensitivity analysis conducted by changing $\sigma$ reveals that for structures with narrow pores, it is important to choose a starting force field that models the correct distance terms in the guest-host interactions.

Finally, the full adsorption isotherms were computed for $\mathrm{CH}_{4}$ in $\mathrm{ABW}$ and FAU and for $\mathrm{CO}_{2}$ in $\mathrm{ABW}, \mathrm{FAU}, \mathrm{SOD}$, and RWY. ABW and FAU were chosen as representative materials for small and large pore structures, respectively. Two additional structures (i.e., SOD and RWY) were chosen for the $\mathrm{CO}_{2}$ case as the constant shift method in changing the epsilon yielded more accurate $\mathrm{CO}_{2} \mathrm{~K}_{\mathrm{H}}$ in these cases. The $\mathrm{CH}_{4}$ and $\mathrm{CO}_{2}$ adsorption isotherms at $T=313 \mathrm{~K}$ are shown in Figures 6 and
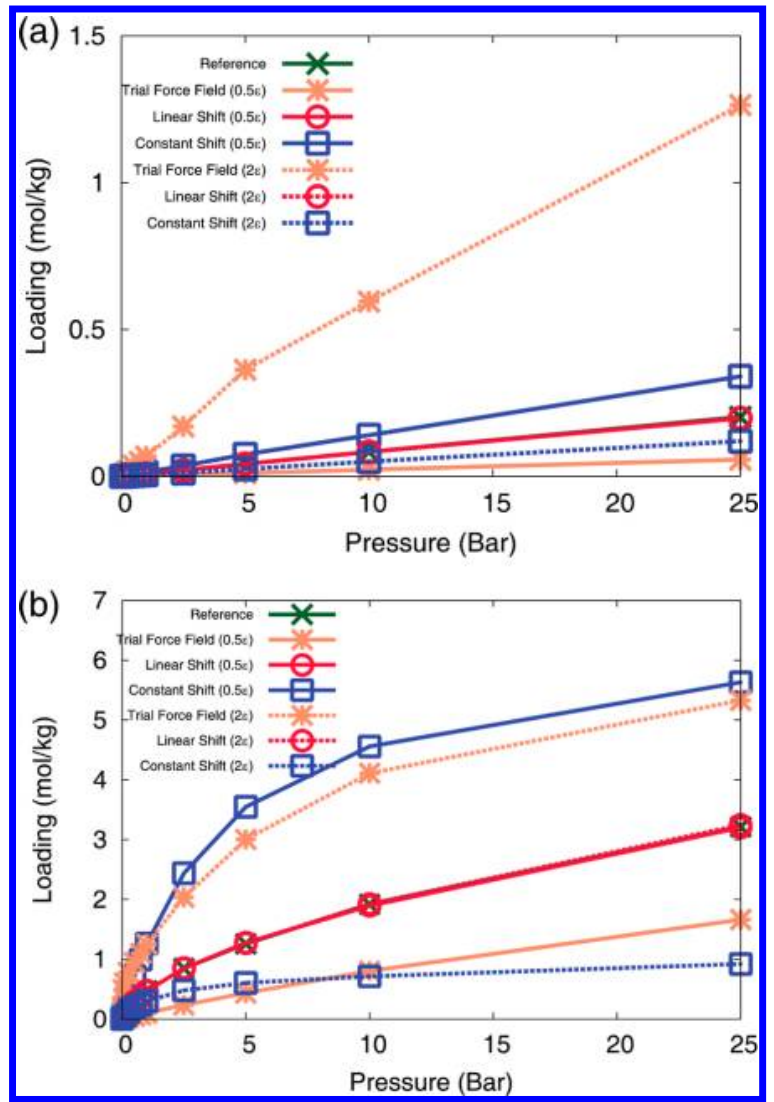

Figure 6. $\mathrm{CH}_{4}$ isotherms at $\mathrm{T}=313 \mathrm{~K}$ for (a) ABW and (b) FAU. The trial force field parameter uses $\varepsilon_{\text {oxygen }}=0.5 \varepsilon=44.68 \mathrm{~K}$ (nondashed) and $\varepsilon_{\text {oxygen }}=2 \varepsilon=178.72 \mathrm{~K}$ (dashed) in the Lennard-Jones potential.

7. The $\mathrm{CH}_{4}$ isotherms (Figure 6) indicate that the linear shift yield isotherm data that are statistically identical to the reference data. The constant shift becomes problematic especially for zeolite FAU, where the large pore size leads to prevalence of secondary binding sites that is incorrectly shifted by the primary binding site energy difference. Accordingly, the empirical linear shift does an adequate job of tempering this effect. For the $\mathrm{CO}_{2}$ isotherms (Figure 7), a similar trend is observed as the zeolites with smaller pore sizes (ABW and SOD) yield good agreement with both linear and constant shifts. For zeolites with larger pores (FAU and RWY), the methodology yields inaccurate data for reasons similar to $\mathrm{CH}_{4}$ in FAU. Unlike the case of $\mathrm{CO}_{2}$ in M-MOF-74 structures, in these zeolites, the lack of strong primary binding sites exacerbates matter as the shifts in the secondary sites are not 

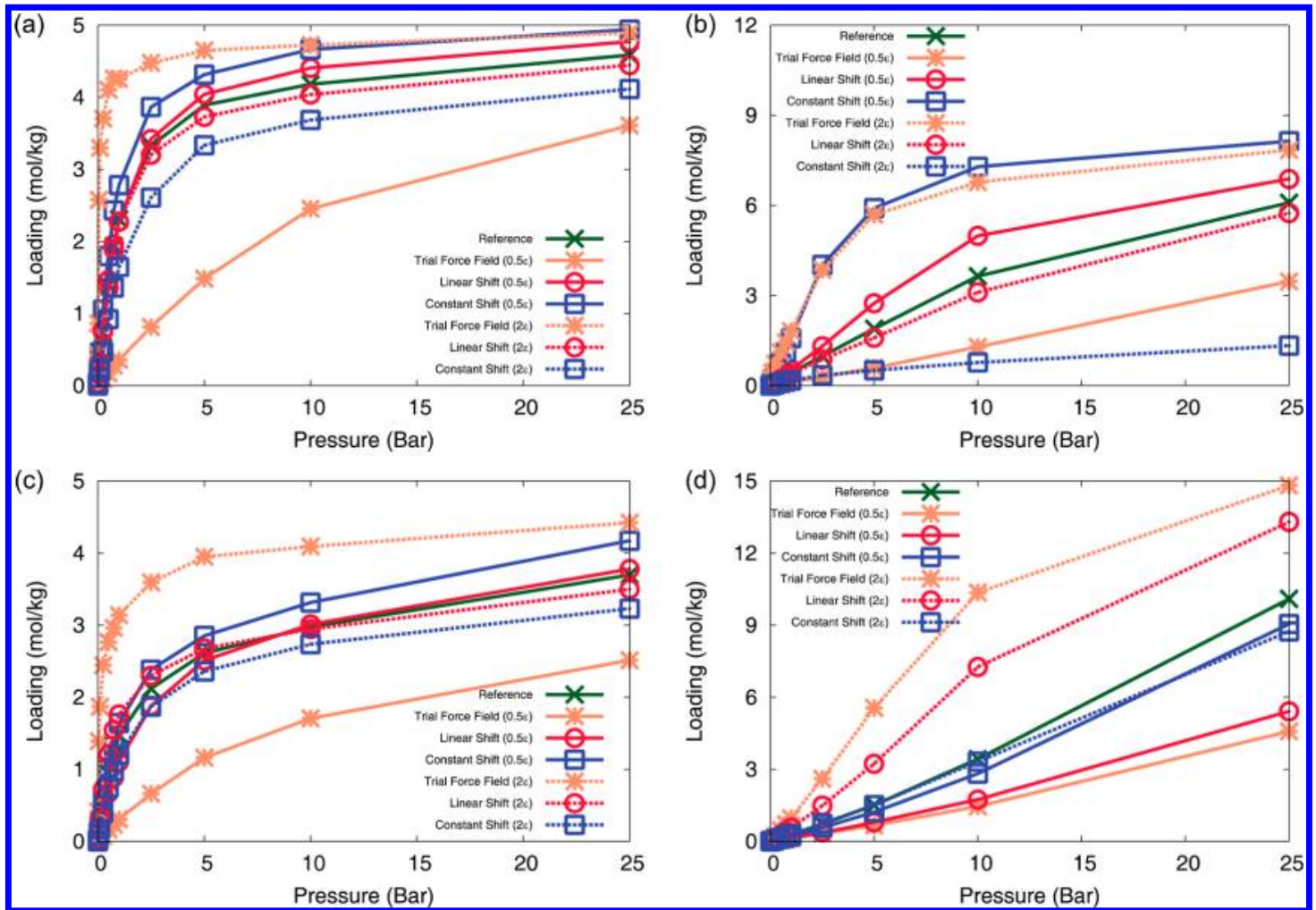

Figure 7. $\mathrm{CO}_{2}$ isotherms at $\mathrm{T}=313 \mathrm{~K}$ for (a) ABW, (b) FAU, (c) SOD, and (d) RWY. Similar to the case for $\mathrm{CH}_{4}$, the trial force field parameter uses $\varepsilon_{\text {oxygen }}=0.5 \varepsilon=44.68 \mathrm{~K}$ (nondashed) and $\varepsilon_{\text {oxygen }}=2 \varepsilon=178.72 \mathrm{~K}$ (dashed) in the Lennard-Jones potential.

only inaccurate but their contributions to the overall adsorption properties are larger. Accordingly, the selection of an accurate force field becomes more crucial in porous materials with weak binding sites and large pore volume when adopting our methodology.

\section{CONCLUSIONS}

We have developed a new methodology that allows simple and efficient, yet accurate, prediction of adsorption properties in porous materials from a judicious number of $\mathrm{ab}$ initio total energy calculations. The methodology involves utilizing a trial force field to obtain reasonably accurate picture of the porous material's adsorption properties and correcting the single point energy values from the quantum mechanical binding energy calculations. We have demonstrated that our methodology leads to accurate adsorption properties for a wide range of guest molecules in porous materials, including $\mathrm{CH}_{4}$ and $\mathrm{CO}_{2}$ inside zeolites and $\mathrm{CO}_{2}$ in Mg-MOF-74 and Fe-MOF-74. Moreover, the predictions made for $\mathrm{N}_{2}$ in V-MOF-74 and Ti-MOF-74 reveal significant uptake of $\mathrm{N}_{2}$ in these two unsynthesized materials. The methodology especially works well for structures that possess strong binding sites as the adsorption properties are largely determined by the low energy regions. Also, for structures with small pores and channels, a trial force field with correct sigma values can lead to more accurate representation of the overall adsorption properties with better performance observed for linear shifts. Finally, the method does poorly for structures with large pore sizes that lack a strong adsorption sites when the trial force field is vastly different from the reference case. In this case, it is imperative to obtain an accurate force field for accurate characterization.

\section{ASSOCIATED CONTENT}

\section{Supporting Information}

Figures showing the stability analysis for $\mathrm{CO}_{2}$ in $\mathrm{Mg}-\mathrm{MOF}-74$; figures showing the $\mathrm{CH}_{4}$ single point energy distributions for eight zeolites. This material is available free of charge via the Internet at http://pubs.acs.org.

\section{AUTHOR INFORMATION}

\section{Corresponding Author}

*E-mail jihankim@kaist.ac.kr (J.K.).

\section{Notes}

The authors declare no competing financial interest.

\section{ACKNOWLEDGMENTS}

J.K. is supported by the KAIST Startup Fund (Project G04130042). B.S. and L.C.L. are supported as part of the Center for Gas Separations Relevant to Clean Energy Technologies, an Energy Frontier Research Center funded by the U.S. Department of Energy, Office of Science, Office of Basic Energy Sciences, under Award DE-SC0001015. K.L. is supported by the U.S. Department of Energy, Office of Basic Energy Sciences, Division of Chemical Sciences, Geosciences and Biosciences, under Award DE-FG02-12ER16362. Work at the Molecular Foundry is also supported by the US Department of Energy, Office of Science, and Office of BES. This research used resources of the National Energy Research Scientific Computing Center (NERSC), which is supported by the Office of Science of the U.S. Department of Energy under Contract DE-AC02-05CH11231. 


\section{REFERENCES}

(1) Rosi, N. L.; Eckert, J.; Eddaoudi, M.; Vodak, D. T.; Kim, J.; O'Keeffe, M.; Yaghi, O. M. Hydrogen storage in microporous metalorganic frameworks. Science 2003, 300, 1127-1129.

(2) Eddaoudi, M.; Kim, J.; Rosi, N.; Vodak, D.; Wachter, J.; O'Keeffe, M.; Yaghi, O. M. Systematic design of pore size and functionality in isoreticular MOFs and their application in methane storage. Science 2002, 295, 469-472.

(3) Seo, J. S.; Whang, D.; Lee, H.; Jun, S.; Oh, J.; Jeon, Y.; Kim, K. A homochiral metal-organic porous material for enantioselective separation and catalysis. Nature 2000, 404, 982-986.

(4) D’Alessandro, D. M.; Smit, B.; Long, J. R. Carbon dioxide capture: prospects for new materials. Angew. Chem., Int. Ed. 2010, 49, 6058-6082.

(5) Sumida, K.; Rogow, D. L.; Mason, J. A.; McDonald, T. M.; Bloch, E. D.; Herm, Z. R.; Bae, T.-H.; Long, J. R. Long Carbon dioxide capture in metal-organic frameworks. Chem. Rev. 2012, 112, 724781.

(6) Getman, R. B.; Bae, Y.-S.; Wilmer, C. E.; Snurr, R. Q. Review and analysis of molecular simulations of methane, hydrogen, and acetylene storage in metal-organic frameworks. Chem. Rev. 2011, 112, 703-723.

(7) Li, J.-R.; Sculley, J.; Zhou, H.-C. Metal-organic frameworks for separations. Chem. Rev. 2011, 112, 869-932.

(8) Wilmer, C. E.; Leaf, M.; Lee, C. Y.; Farha, O. K.; Hauser, B. G.; Hupp, J. T.; Snurr, R. Q. Large-scale screening of hypothetical metalorganic frameworks. Nat. Chem. 2012, 4, 83-89.

(9) Keskin, S.; Sholl, D. S. Efficient methods for screening of metal organic framework membranes for gas separations using atomically detailed models. Langmuir 2009, 25, 11786-11795.

(10) Lin, L.-C.; Berger, A.; Martin, R.; Kim, J.; Swisher, J.; Jariwala, K.; Rycroft, C.; Bhown, A.; Deem, M.; Haranczyk, M.; Smit, B. In silico screening of carbon-capture materials. Nat. Mater. 2012, 11, 633-641.

(11) Krishna, R.; Van Baten, J. M. In silico screening of metalorganic frameworks in separation applications. Phys. Chem. Chem. Phys. 2011, 13, 10593-10616.

(12) Kim, J.; Lin, L.-C.; Swisher, J. A.; Haranczyk, M.; Smit, B. Predicting large $\mathrm{CO}_{2}$ adsorption in aluminosilicate zeolites for postcombustion carbon dioxide capture. J. Am. Chem. Soc. 2012, 134, 18940-18943.

(13) First, E. L.; Gounaris, C. E.; Wei, J.; Floudas, C. A. Computational characterization of zeolite porous networks: an automated approach. Phys. Chem. Chem. Phys. 2011, 13, 1733917358.

(14) Kim, J.; Maiti, A.; Lin, L.-C.; Stolaroff, J. K.; Smit, B.; Aines, R. D. New materials for methane capture from dilute and mediumconcentration sources. Nat. Commun. 2013, 4, 1694.

(15) Fang, H.; Kamakoti, P.; Zang, J.; Cunday, S.; Paur, C.; Ravikovitch, P.; Sholl, D. Prediction of $\mathrm{CO}_{2}$ adsorption properties in zeolites using force fields derived from periodic dispersion-corrected DFT calculations. J. Phys. Chem. C 2012, 116, 10692-10701.

(16) Fang, H.; Kamakoti, P.; Ravikovitch, P.; Aronson, M.; Paur, C.; Sholl, D. First principles derived transferable force fields for $\mathrm{CO}_{2}$ adsorption in Na-exchanged cationic zeolites. Phys. Chem. Chem. Phys. 2013, 15, 12882-12894.

(17) McDaniel, J.; Yu, K.; Schmidt, J. R. Ab initio, physically motivated force fields for $\mathrm{CO}_{2}$ adsorption in zeolitic imidazolate frameworks. J. Phys. Chem. C 2012, 116, 1892-1903.

(18) Han, S. S.; Kim, D.; Jung, D.; Cho, S.; Choi, S.-H.; Jung, Y. Accurate $\mathrm{ab}$ initio-based force field for predictive $\mathrm{CO}_{2}$ uptake simulations in MOFs and ZIFs: Development and applications for MTV-MOFs. J. Phys. Chem. C 2012, 116, 20254-20261.

(19) Dzubak, A.; Lin, L.-C.; Kim, J.; Swisher, J. A.; Poloni, R.; Maximoff, S. N.; Smit, B.; Gagliardi, L. Ab initio carbon capture in open-site metal-organic frameworks. Nat. Chem. 2012, 4, 810-816.

(20) Chen, L.; Morrison, C. A.; Düren, T. Improving predictions of gas adsorption in metal-organic frameworks with coordinatively unsaturated metal sites: Model potentials, ab initio parameterization, and GCMC simulations. J. Phys. Chem. C 2012, 116, 18899-18909.
(21) Sillar, K.; Hofmann, A.; Sauer, J. Ab initio study of hydrogen adsorption in MOF-5. J. Am. Chem. Soc. 2009, 131, 4143-4150.

(22) Chui, S.; Lo, S.; Charmant, J.; Orpen, A.; Williams, I. A chemically functionalizable nanoporous material $\left[\mathrm{Cu}_{3}(\mathrm{TMA})_{2}\left(\mathrm{H}_{2} \mathrm{O}\right)_{3}\right]_{\mathrm{n}}$. Science 1999, 283, 1148-1150.

(23) Millward, A. R.; Yaghi, O. M. Metal-organic frameworks with exceptionally high capacity for storage of carbon dioxide at room temperature. J. Am. Chem. Soc. 2005, 127, 17998-17999.

(24) Dietzel, P.; Besikiotis, V.; Blom, R. Application of metalorganic frameworks with coordinatively unsaturated metal sites in storage and separation of methane and carbon dioxide. J. Mater. Chem. 2009, 19, 7362-7370.

(25) Grajciar, L. S.; Buldsky, O.; Nachtigall, P. Water adsorption on coordinatively unsaturated sites in CuBTC MOF. J. Phys. Chem. Lett. 2010, 1, 3354-3359.

(26) McDonald, T. M.; Lee, W.; Mason, J.; Wiers, B.; Hong, C.; Long, J. Capture of carbon dioxide from air and flue gas in the alkylamine-appended metal-organic framework mmen- $\mathrm{Mg}_{2}$ (dobpdc). J. Am. Chem. Soc. 2012, 134, 7056-7065.

(27) Kim, J.; Martin, R.; Rübel, O.; Haranczyk, M.; Smit, B. Highthroughput characterization of porous materials using graphics processing units. J. Chem. Theory Comput. 2012, 8, 1684-1693.

(28) Campañá, C.; Mussard, B.; Woo, T. K. Electrostatic potential derived atomic charges for periodic systems using a modified error functional. J. Chem. Theory Comput. 2009, 5, 2866-2878.

(29) Giannozzi, P.; Baroni, S.; Bonini, N.; Calandra, M.; Car, R.; Cavazzoni, C.; Ceresoli, D.; Chiarotti, G.; Cococcioni, M.; Dabo, I.; et al. QUANTUM ESPRESSO: a modular and open-source software project for quantum simulations of materials. J. Phys.: Condens. Matter 2009, 21, 395502.

(30) Mason, J. A.; Sumida, K.; Herm, Z.; Krishna, R.; Long, J. R. Evaluating metal-organic frameworks for post-combustion carbon dioxide capture via temperature swing adsorption. Energy Environ. Sci. 2011, 4, 3030-3040.

(31) Lin, L.-C.; Kim, J.; Kong, X.; Scott, E.; McDonald, T. M.; Long, J. R; Reimer, J. A.; Smit, B. Understanding $\mathrm{CO}_{2}$ dynamics in metalorganic frameworks with open metal sites. Angew. Chem., Int. Ed. 2013, $52,4410-4413$.

(32) Dion, M.; Rydberg, H.; Schröder, E.; Langreth, D. C.; Lundqvist, B. I. Van der Waals density functional for general geometries. Phys. Rev. Lett. 2004, 92, 246401.

(33) Lee, K.; Murray, E. D.; Kong, L.; Lundqvist, B. I.; Langreth, D. C. Higher-accuracy van der Waals density functional. Phys. Rev. B 2010, 82, 081101.

(34) Dudarev, S. L.; Botton, G. A.; Savrasov, S. Y.; Humphreys, C. J.; Sutton, A. P. Electron-energy-loss spectra and the structural stability of nickel oxide: An LSDA+ U study. Phys. Rev. B 1998, 57, 1505-1509.

(35) Kresse, G.; Furthmüller, J. Efficient iterative schemes for $a b$ initio total-energy calculations using a plane-wave basis set. Phys. Rev. B 1996, 54, 11169.

(36) Märcz, M.; Johnsen, R; Dietzel, P.; Fjellvåg, H. The iron member of the CPO-27 coordination polymer series: Synthesis, characterization, and intriguing redox properties. Microporous Mesoporous Mater. 2012, 157, 62-74.

(37) Lee, K.; Isley, W.; Dzubak, A.; Verma, P.; Stoneburner, S.; Lin, L.-C.; Howe, J.; Bloch, E.; Reed, D.; Hudson, M.; et al. Design of a metal-organic framework with enhanced back bonding for the separation of $\mathrm{N}_{2}$ and $\mathrm{CH}_{4}$. J. Am. Chem. Soc. 2014, 136, 698-704.

(38) http://www.iza-structure.org/databases/, accessed August 1, 2013.

(39) García-Pérez, E.; Parra, J. B.; Ania, C. O.; García-Sánchez, A.; Baten, J. M.; Krishna, R.; Dubbeldam, D.; Calero, S. A computational study of $\mathrm{CO}_{2}, \mathrm{~N}_{2}$, and $\mathrm{CH}_{4}$ adsorption in zeolites. Adsorption 2007, 13, $469-476$.

(40) Willems, T.; Rycroft, C.; Kazi, M.; Meza, J.; Haranczyk, M. Algorithms and tools for high-throughput geometry-based analysis of crystalline porous materials. Microporous Mesoporous Mater. 2012, 149, 134-141. 